\title{
SAMPLING 2-D SIGNALS ON A UNION OF LATTICES THAT INTERSECT ON A LATTICE
}

\author{
Jayakrishnan Unnikrishnan* \\ School of Computer and Communication Sciences \\ Ecole Polytechnique Fédérale de Lausanne (EPFL) \\ CH-1015 Lausanne, Switzerland \\ jay.unnikrishnan@epfl.ch
}

Matthew A. Prelee

\author{
EECS Department \\ University of Michigan \\ Ann Arbor, MI 48109 \\ mprelee@umich.edu
}

\begin{abstract}
This paper presents new sufficient conditions under which a field (or image) can be perfectly reconstructed from its samples on a union of two lattices that share a common coarse lattice. In particular, if samples taken on the first lattice can be used to reconstruct a field bandlimited to some spectral support region, and likewise samples taken on the second lattice can reconstruct a field bandlimited to another spectral support region, then under certain conditions, a field bandlimited to the union of these two spectral regions can be reconstructed from its samples on the union of the two respective lattices. These results generalize a previous perfect reconstruction theorem for Manhattan sampling, where data is taken at high density along evenly spaced rows and columns of a rectangular grid. Additionally, a sufficient condition is given under which the Landau lower bound is achieved.
\end{abstract}

Index Terms - Image sampling, sampling methods.

\section{INTRODUCTION}

Manhattan sampling is a new approach to sampling two dimensional fields (i.e. images) where data is taken densely along evenly spaced rows and columns. An example of a Manhattan grid is shown in Fig.1, where there are $k_{1}=4$ samples between each column and $k_{2}=3$ samples between each row. This is a special case of cutset sampling, which has been used to good effect in both lossy and lossless image compression, especially, for bilevel images [1-3]. Manhattan sampling has also been proposed as a new approach to sampling grayscale images [4,5]. Finally, Manhattan sampling is useful in wireless sensor network applications where the goal is to estimate a two-dimensional field. If sensors are deployed on a Manhattan grid, as opposed to random placement, then the energy costs of data transmission tends tend to be much smaller [6]. Such "Manhattan networks" can be used to efficiently solve the problem of RSS-based source localization [7].

Manhattan sampling can be viewed as sampling on the union of two lattices: one lattice is dense in the horizontal direction and coarse in the vertical direction, while the other lattice is coarse in the horizontal direction and dense in the vertical direction. Recently, a sampling theorem for Manhattan grids showed that a field can be recovered from its Manhattan grid samples when its spectrum is supported on the union of rectangular Nyquist regions corresponding to the spacing of the two rectangular lattices [8]. An example of such a cross-shaped region is shown in Figure 2(a). This region is maximal in the Landau sense, i.e. the Lebesgue measure of the union

\footnotetext{
* This work was supported by ERC Advanced Investigators Grant no. 247006 .
}

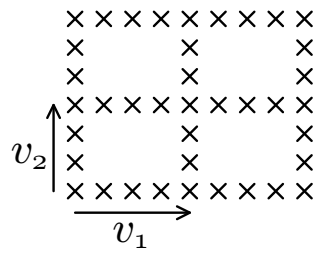

(a) Manhattan grid

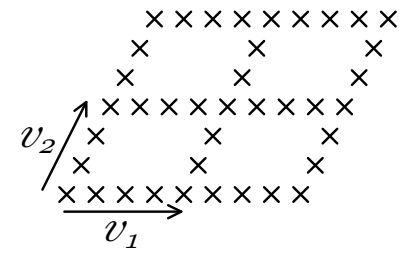

(b) Slanted Manhattan grid
Fig. 1. Examples of a union of two lattices that share a common coarse lattice with $k_{1}=4$ and $k_{2}=3$.

of Nyquist regions is exactly the sampling density of the Manhattan sampling set [9].

Sampling theorems for a single lattice in multiple dimensions have long been studied [10] (see also [11, p. 72], [12, p. Chap. 3], [13, p. 43]). Recently, Venkataramani and Bresler obtained results for sampling on unions of shifted lattices in one dimension $[14,15]$, finding conditions under which the sampling density could reach or become arbitrary close to the Landau bound. Additionally, Behmard and Faridani studied sampling on unions of shifted lattices in two dimensions, giving "compatibility conditions" such that perfect recovery was possible $[16,17]$. However, such conditions are not satisfied by Manhattan sampling and the spectral support region consisting of the union of Nyquist regions. They also did not give conditions under which the Landau lower bound was reached.

A limiting case of Manhattan sampling is when the density of samples along the grid lines can be increased arbitrarily. Sampling theorems and reconstruction methods for this limiting scenario were studied in [18] and [19]. The primary application addressed in these works was the sampling of spatially bandlimited fields using mobile sensors [20]. In these works, the spectra of such fields were required to satisfy several conditions, among them being the condition that the spectral support be convex. Again, convexity is not satisfied by a spectral support consisting of the union of Nyquist regions.

In this work, we generalize the result of [8] to slanted Manhattan grids, which is the union of two lattices that share a common coarse lattice, as shown in Fig.1. We find new sufficient conditions under which a two-dimensional field can be perfectly reconstructed from its samples on a slanted Manhattan grid. We also generalize the limiting case result of [18] and [19] to arbitrary non-convex spectral regions. We are particularly concerned with conditions where the image spectrum explicitly satisfies the Landau bound. Reconstruction conditions are given in Section 2.1, and the Landau condition is given in Section 3. We conclude our findings in Section 4. 


\section{PROBLEM DESCRIPTION AND PROPOSED SOLUTION}

We begin by describing some notation. Let $x: \mathbb{R}^{2} \rightarrow \mathbb{C}$ be a square-integrable field (image) with corresponding Fourier transform $X$ given by

$$
X(f)=\int_{\mathbb{R}^{2}} x(t) \exp (-\mathrm{i} 2 \pi\langle t, f\rangle) d t .
$$

Let $\Omega$ be a compact subset of $\mathbb{R}^{2}$. For $x \in L^{2}\left(\mathbb{R}^{2}\right)$, we say that $x$ (or $X$ ) is bandlimited to $\Omega$ if the spectrum $X$ is supported on $\Omega$, i.e. $X(f)=0, \forall f \notin \Omega$. Let $L$ denote a countable set of points in the plane. We say $L$ is a sampling set for region $\Omega$ if all functions $x$ bandlimited to $\Omega$ can be uniquely reconstructed from their values $\{x(t), t \in L\}$. Let $D(L)$ denote the density of a sampling set $L$ in two-dimensions defined as

$$
D(L)=\liminf _{r \rightarrow \infty} \inf _{y \in \mathbb{R}^{2}} \frac{\#\left(L \cap B_{r}(y)\right)}{4 r^{2}}
$$

where \# denotes cardinality and $B_{r}(y):=\left\{z \in \mathbb{R}^{2}:\|z-y\|_{\infty} \leq\right.$ $r\}$ is a square of side $2 r$ centered at $y$. Landau showed that a necessary condition for $L$ to be a sampling set for $\Omega$ is that $D(L) \geq|\Omega|$ [9], where $|\Omega|$ denotes the Lebesgue measure of the set $\Omega$. We say that $L$ achieves the Landau bound if $D(L)=|\Omega|$.

In this paper, we are primarily concerned with sampling sets that are lattices or unions of lattices. Let $v_{1}, v_{2} \in \mathbb{R}^{2}$ be linearly independent vectors that generate a coarse lattice $L_{C}=\left\{m v_{1}+n v_{2}\right.$ : $m, n \in \mathbb{Z}\}$. Let $u_{1}, u_{2}$ satisfy

$$
\left\langle u_{i}, v_{j}\right\rangle=\delta_{i j}
$$

so that $\left\{u_{1}, u_{2}\right\}$ generate the reciprocal lattice $L_{C}^{\perp}$. Let $L_{1}\left(L_{2}\right)$ denote a lattice generated by the vectors $\left\{\frac{v_{1}}{k_{1}}, v_{2}\right\}\left(\left\{v_{1}, \frac{v_{2}}{k_{2}}\right\}\right)$, where $k_{1}, k_{2} \in \mathbb{Z}_{+} \backslash\{0\}$ and $\max \left\{k_{1}, k_{2}\right\}>1$. Let $\Omega_{1}\left(\Omega_{2}\right)$ be a compact subset of $\mathbb{R}^{2}$ such that $L_{1}\left(L_{2}\right)$ forms a sampling lattice for images bandlimited to $\Omega_{1}\left(\Omega_{2}\right)$. Let $\Omega:=\Omega_{1} \cup \Omega_{2}$ and $S:=\Omega_{1} \cap \Omega_{2}$. In addition, we assume that the boundaries of $\Omega_{i}$ have zero Lebesgue measure.

We study the problem of sampling on $M:=L_{1} \cup L_{2}$, the union of two lattices that intersect at a common coarse lattice $L_{C}$. This includes the Manhattan grid shown in Fig.1(a) or the more general slanted Manhattan grid shown in Fig.1(b). We are interested in fields bandlimited to the union of frequency support regions $\Omega=\Omega_{1} \cup \Omega_{2}$. In particular, we try to answer the following two questions:

When does $M$ form a sampling lattice for $\Omega$ ?

and

When does $M$ achieve the Landau bound for $\Omega$ ?

The answer to (2) was answered previously in [8] for rectangular Manhattan grids when $\Omega_{1}$ and $\Omega_{2}$ were the rectangular Nyquist regions centered at the origin, corresponding to lattices $L_{1}$ and $L_{2}$, respectively. Although it was not explicitly stated in the previous work, it can be shown that the Landau bound is achieved in this case. The main focus of this paper is to generalize this result to the union of two lattices that share a common coarse lattice, and for more general frequency support regions than the rectangular Nyquist regions.

\subsection{Preliminaries}

First, let us consider the two sampling lattices $L_{1}$ and $L_{2}$ separately and see their effects in the frequency domain. It is clear that the reciprocal lattice $L_{1}^{\perp}$ of $L_{1}$ is generated by the vectors $\left\{k_{1} u_{1}, u_{2}\right\}$ and that the reciprocal lattice $L_{2}^{\perp}$ of $L_{2}$ is generated by the vectors $\left\{u_{1}, k_{2} u_{2}\right\}$. Thus the sampled spectrum from $L_{1}$ and $L_{2}$ are respectively given by

$$
\begin{aligned}
& X_{1}(f)=\sum_{i, j \in \mathbb{Z}} X\left(f-i k_{1} u_{1}-j u_{2}\right)=\sum_{f^{\prime} \in L_{1}^{\perp}} X\left(f+f^{\prime}\right), \\
& X_{2}(f)=\sum_{i, j \in \mathbb{Z}} X\left(f-i u_{1}-j k_{2} u_{2}\right)=\sum_{f^{\prime} \in L_{2}^{\perp}} X\left(f+f^{\prime}\right) .
\end{aligned}
$$

These equations are the basis of results in this paper.

\subsection{Sufficiency via aliasing condition}

The following proposition gives a simple sufficient condition for (2).

Proposition 2.1 (Spectral Reconstruction via Aliasing Condition:). For two sets $B_{1}$ and $B_{2}$, define $B_{1}+B_{2}=\left\{b_{1}+b_{2}: b_{1} \in B_{1}, b_{2} \in\right.$ $\left.B_{2}\right\}$. Suppose

$$
\left(\Omega_{2}+L_{1}^{\perp}\right) \cap \Omega_{1}=S .
$$

Then the set $M=L_{1} \cup L_{2}$ forms a sampling set for $\Omega_{1} \cup \Omega_{2}$.

Proof. Assume $\left(\Omega_{2}+L_{1}^{\perp}\right) \cap \Omega_{1}=S$ is true. Let $f \in \Omega_{1} \backslash S$ be arbitrary. If there exists $f^{\prime} \in L_{1}^{\perp}$ such that $f+f^{\prime} \in \Omega_{2}$, then $f \in\left(\Omega_{2}+L_{1}^{\perp}\right)$ and thus

$$
f \in\left(\Omega_{2}+L_{1}^{\perp}\right) \cap \Omega_{1} \backslash S
$$

which contradicts (6). Thus for all $f^{\prime} \in L_{1}^{\perp}$ we must have $f+f^{\prime} \notin$ $\Omega_{2}$. Hence for $f \in \Omega_{1} \backslash S$ the sampled spectrum of (4) satisfies

$$
X_{1}(f)=\sum_{\substack{f^{\prime} \in L_{1}^{\perp} \\ f+f^{\prime} \in \Omega_{1} \backslash S}} X\left(f+f^{\prime}\right) .
$$

Since $L_{1}$ forms a sampling lattice for $\Omega_{1}$ it further follows that

$$
X_{1}(f)=X(f), \text { for all } f \in \Omega_{1} \backslash S
$$

and thus the portion of the spectrum in $\Omega_{1} \backslash S$ can be decoded first. Now consider (5), which can be rewritten under the given bandlimitation assumptions as

$$
X_{2}(f)=\sum_{\substack{f^{\prime} \in L_{2}^{\perp} \\ f+f^{\prime} \in \Omega_{1} \backslash S}} X\left(f+f^{\prime}\right)+\sum_{\substack{f^{\prime} \in L^{\frac{1}{2}} \\ f+f^{\prime} \in \Omega_{2}}} X\left(f+f^{\prime}\right)
$$

The first term is known since $\Omega_{1} \backslash S$ has already been decoded, and thus can be replaced by $X\left(f+f^{\prime}\right)=X_{1}\left(f+f^{\prime}\right)$. Furthermore, the second term is exactly $X(f)$ for $f \in \Omega_{2}$, since $L_{2}$ is a sampling lattice for $\Omega_{2}$ by assumption. Thus, for $f \in \Omega_{2}, X(f)$ can be recovered by simply subtracting the known first term from $X_{2}(f)$. The entire reconstruction procedure is thus given by

$$
X(f)= \begin{cases}X_{1}(f), & f \in \Omega_{1} \backslash S \\ X_{2}(f)-\sum_{\substack{f^{\prime} \in L_{2}^{\perp} \\ f+f^{\prime} \in \Omega_{1} \backslash S}} X_{1}\left(f+f^{\prime}\right), & f \in \Omega_{2} .\end{cases}
$$

A special case of this result is presented next. 


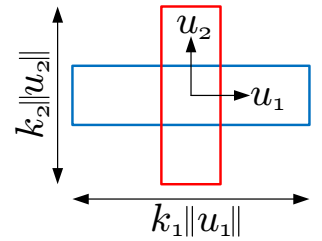

(a) Satisfies conditions in [8] and Propositions 2.1, 2.2, 2.4

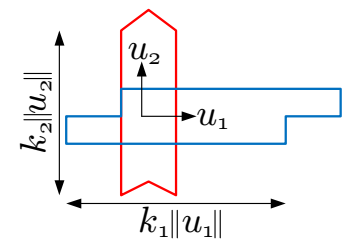

(b) Satisfies Propositions 2.2 (implies satisfying Prop. 2.1).

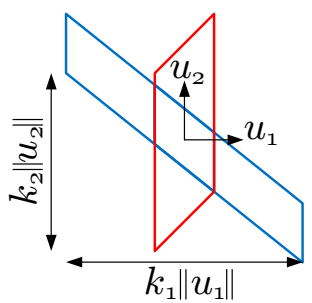

(c) Satisfies Proposition 2. but not Props. 2.2 or 2.4 .

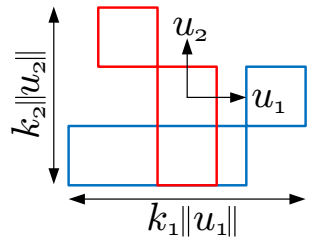

(d) Satisfies Proposition 2.4 but not Propositions 2.1 or 2.2

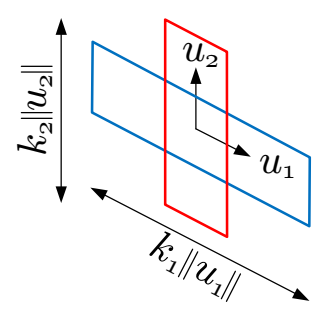

(e) Satisfies Proposition 2.2.

Fig. 2. Examples of supports of spectra that can be reconstructed using results presented in this paper. (a-d) can be recovered from samples on the rectangular Manhattan grid in Fig.1(a) and (e) can be recovered from samples on the slanted Manhattan grid in Fig.1(b). The blue curves indicate the boundary of $\Omega_{1}$ and red curves that of $\Omega_{2}$.

Proposition 2.2 (Aliasing conditions for spectra in strips:). Let $\ell_{i}$ denote any line parallel to $u_{i}$. Suppose $\Omega_{1}\left(\Omega_{2}\right)$ is a set contained within the strip between lines $\ell_{1}$ and $\ell_{1}+u_{2}\left(\ell_{2}\right.$ and $\left.\ell_{2}+u_{1}\right)$ such that $S=\Omega_{1} \cap \Omega_{2}$ is the entire region contained inside a parallelogram bounded by $\ell_{1}, \ell_{1}+u_{2}, \ell_{2}, \ell_{2}+u_{1}$. Then $M=L_{1} \cup L_{2}$ forms a sampling set for $\Omega$.

Sketch of proof. From the geometry of the sets it can be seen that

$$
\left(\Omega_{2}+L_{1}^{\perp}\right) \cap \Omega_{1}=\left(S+L_{1}^{\perp}\right) \cap \Omega_{1} .
$$

However since $L_{1}$ is a sampling lattice for $\Omega_{1}$ it follows that the second set above must equal $S$. The conclusion then follows from Proposition 2.1.

The result of sampling on the union of two rectangular lattices considered in [8] follows immediately from the above result.

\subsection{Sufficiency via graph condition}

In some applications like mobile sensing [18], the over-sampling factors $k_{1}$ and $k_{2}$ can be increased without too much additional cost. Let $P$ be a parallelogram with two sides parallel to $u_{1}$ of magnitude $k_{1}\left\|u_{1}\right\|$ and two sides parallel to $u_{2}$ of magnitude $k_{2}\left\|u_{2}\right\|$. Suppose $k_{i}$ are large enough so that $\Omega$ is contained within some shifted version of $P$. In such a scenario, the sampled spectra in (4) and (5) are aliased only in one direction and thus satisfy

$$
X_{1}(f)=\sum_{j \in \mathbb{Z}} X\left(f-j u_{2}\right), f \in \Omega
$$

and

$$
X_{2}(f)=\sum_{i \in \mathbb{Z}} X\left(f-i u_{1}\right), f \in \Omega .
$$

In this case, a different sufficient condition for (2) can be obtained via a condition on a graph. We need the following lemma proved using a generalization of the technique used in [18, Thm 2.4].

Lemma 2.3. Let $L=\left\{m u_{1}+n u_{2}: m, n \in \mathbb{Z}\right\}$ denote the twodimensional lattice generated by the vectors $u_{1}$ and $u_{2}$, and let $V$ denote a finite subset of $L$. By an abuse of notation we use $G=$ $(V, E)$ to denote a graph whose vertices correspond to the elements of $V$. Vertices $f_{1}, f_{2} \in V$ are connected by an edge if $f_{1}-f_{2}$ is an integer multiple of $u_{1}$ or $u_{2}$. Furthermore, for $f_{1}, f_{2} \in V$ we say that $f_{1}$ and $f_{2}$ are connected by a horizontal edge if $f_{1}-f_{2}$ is an integer multiple of $u_{1}$. In this case we also say that $f_{1}$ and $f_{2}$ are horizontal neighbors. Similarly we say that $f_{1}$ and $f_{2}$ are connected by a vertical edge and that $f_{1}$ and $f_{2}$ are vertical neighbors if $f_{1}-f_{2}$ is an integer multiple of $u_{2}$. We use $\mathcal{N}_{1}^{G}(f)\left(\mathcal{N}_{2}^{G}(f)\right)$ to denote the set of all vertical (horizontal) neighbors of $f$.

Suppose that there is a value $X(f)$ associated with all vertices $f \in V$, such that for each $f \in V$ we are given consistent (i.e., they admit at least one solution) linear equations of the form

$$
X_{1}(f)=\sum_{f^{\prime} \in \mathcal{N}_{2}^{G}(f)} X\left(f^{\prime}\right), \quad f \in V,
$$

and

$$
X_{2}(f)=\sum_{f^{\prime} \in \mathcal{N}_{1}^{G}(f)} X\left(f^{\prime}\right), \quad f \in V .
$$

Suppose further that the graph $G$ does not contain any cycle with alternating horizontal and vertical edges. Then there is a unique solution to the equations of (10) and (11).

Proof. We prove this statement by induction on $|V|$. Clearly if $|V|=1$ the statement is true since in this case equation (10) becomes $X_{1}(f)=X(f)$ for $f \in V$.

Suppose that the claim is true for all choices of $V$ with $|V|=$ $N-1$. We will now show that the statement is true for all choices of $V$ with $|V|=N$. Consider any maximal-length alternating path $P$ in graph $G$ alternating between horizontal and vertical edges such that no two adjacent edges traversed in the path are both horizontal or both vertical. Such a maximal path must exist because $V$ is finite and $G$ does not have alternating cycles. Pick any end-point vertex $f^{\prime}$ of $P$. Assume without loss of generality that the edge in $P$ incident on $f^{\prime}$ is horizontal. Then $f^{\prime}$ does not have vertical neighbors because otherwise the path would not have been maximal. Since $f^{\prime}$ does not have vertical neighbors (10) becomes $X_{1}\left(f^{\prime}\right)=X\left(f^{\prime}\right)$. Thus $X\left(f^{\prime}\right)$ can be uniquely decoded. Let $G^{\prime}=\left(V^{\prime}, E^{\prime}\right)$ be the subgraph of $G$ obtained by removing from $G$ the vertex $f^{\prime}$ and all edges incident at $f^{\prime}$. Clearly, $G^{\prime}$ has $N-1$ vertices and does not contain any cycle with alternating horizontal and vertical edges. For each $f \in V^{\prime}$ define

$$
\begin{aligned}
& Y_{1}(f)= \begin{cases}X_{1}(f)-X\left(f^{\prime}\right), & \text { if } f \in \mathcal{N}_{2}^{G}\left(f^{\prime}\right), \\
X_{1}(f), & \text { otherwise, }\end{cases} \\
& Y_{2}(f)= \begin{cases}X_{2}(f)-X\left(f^{\prime}\right), & \text { if } f \in \mathcal{N}_{1}^{G}\left(f^{\prime}\right), \\
X_{2}(f), & \text { otherwise. }\end{cases}
\end{aligned}
$$


Combining with (10) and (11) it is clear that the following are true:

$$
Y_{1}(f)=\sum_{\tilde{f} \in N_{2}^{G^{\prime}}(f)} X(\tilde{f}), \quad f \in V^{\prime},
$$

and

$$
Y_{2}(f)=\sum_{\tilde{f} \in N_{1}^{G^{\prime}}(f)} X(\widetilde{f}), \quad f \in V^{\prime} .
$$

Thus $G^{\prime}$ satisfies all the conditions of the induction assumption and hence it follows that all vertices in $G^{\prime}$ can be uniquely decoded. Thus all vertices in $G$ can be uniquely decoded. Since this holds for all values of $N$, it follows by the principle of mathematical induction that the lemma is proved for all choices of $V$.

In order to apply the lemma we need some notation and terminology. For every $f \in \Omega$ consider the set of frequencies

$$
A_{f}:=\left\{f^{\prime} \in \Omega: f^{\prime}-f=a u_{1}+b u_{2} \text { for some } a, b \in \mathbb{Z}\right\} .
$$

Let $G_{f}$ denote a graph with vertices representing the entries of $A_{f}$. In $G_{f}$ we say that vertices corresponding to frequencies $f_{1}, f_{2}$ are connected by an edge if $f_{1}-f_{2}$ is an integer multiple of $u_{1}$ or $u_{2}$, and define horizontal and vertical edges and neighbors as in the statement of Lemma 2.3. The following result is immediate from the lemma.

Proposition 2.4 (Spectral Reconstruction via Graph Condition). Suppose the following conditions are true.

1. The sampled spectra of (4) and (5) satisfy (8) and (9).

2. For every $f \in \Omega$, the graph $G_{f}$ does not contain any cycle with alternating horizontal and vertical edges. Equivalently, this means that all cycles in $G_{f}$ contain at least one pair of consecutive horizontal edges or one pair of consecutive vertical edges.

Then the set $M:=L_{1} \cup L_{2}$ forms a sampling set for $\Omega=\Omega_{1} \cup \Omega_{2}$.

Proof. It is easy to see that conditions of Lemma 2.3 are satisfied by $A_{f}$ for all $f \in \Omega$. It follows that $X(f)$ can be uniquely identified for all $f \in \Omega$, and thus $M$ is a sampling set for $\Omega$.

We remark that the result of Proposition 2.4 holds for all $\Omega$ that satisfy the conditions of the proposition, whether or not it can be expressed as a union of $\Omega_{1}$ and $\Omega_{2}$.

It is possible to design an iterative reconstruction algorithm for reconstructing fields that satisfy the conditions of Proposition 2.4 by following the steps in the proof. However, we do not include the details due to lack of space. A weakness of Proposition 2.4 is that a graph $G_{f}$ must be formed for each $f \in \Omega$ and then a set of equations must be solved. In general, this is difficult because $\Omega$ is an uncountable set, but in practice there are often subsets of $\Omega$ that have identical graph structures $G_{f}$, and thus groups of frequencies can be recovered simultaneously. An example of such a case is illustrated in Figure 2(d). We believe that it is possible to generalize Proposition 2.4 to solving a finite number of graph problems, instead of having to solve a separate graph problem for every $f \in \Omega$.

Two examples of supports of spectra that satisfy conditions of Proposition 2.1 are shown in Figures 2(a,b,c,e). Note that only Figures 2(a,b,e) satisfy the conditions for Proposition 2.2. We also note that Figure 2(c) does not satisfy the conditions of Propositions 2.2 or 2.4, despite satisfying Proposition 2.1. Now consider a spectrum supported on the region shown in Figure 2(e). It can be seen that that this spectrum satisfies the conditions of Proposition 2.4 but not of Proposition 2.1. Thus examples of Figures 2(c) and 2(e) suggest that Propositions 2.4 and 2.1 are both not implied by the other.

\section{ACHIEVING THE LANDAU BOUND}

We now present a useful lemma that gives a condition under which achieving the Landau bound is preserved when sampling on a union of lattices that share a common coarse lattice, thus providing some insight to question (3).

Lemma 3.1. For $i=1,2$, let $L_{i}$ be a sampling set for $\Omega_{i}$ that achieves the Landau bound, and suppose that $L_{C}=L_{1} \cap L_{2}$ forms a sampling set for fields bandlimited to the intersection $S=\Omega_{1} \cap \Omega_{2}$. If the set $M=L_{1} \cup L_{2}$ forms a sampling set for $\Omega_{1} \cup \Omega_{2}$, then $M$ achieves the Landau bound.

Proof. Using $D(L)$ to denote density of a sampling set $L$, we have

$$
\begin{aligned}
D(M) & =D\left(L_{1}\right)+D\left(L_{2}\right)-D\left(L_{C}\right) \\
& =\left|\Omega_{1}\right|+\left|\Omega_{2}\right|-D\left(L_{C}\right) \\
& \leq\left|\Omega_{1}\right|+\left|\Omega_{2}\right|-\left|\Omega_{1} \cap \Omega_{2}\right| \\
& =\left|\Omega_{1} \cup \Omega_{2}\right| .
\end{aligned}
$$

The first step follows from the definition of $M$, and the second relation follows because $L_{i}$ achieves the Landau bound. Since $L_{C}$ is a sampling lattice for $S=\Omega_{1} \cap \Omega_{2}$ by assumption, the third step follows from the fact that $D\left(L_{C}\right) \geq\left|\Omega_{1} \cap \Omega_{2}\right|$. The final relation follows from elementary set theory. Since it was originally assumed that $M$ is a sampling set for $\Omega_{1} \cup \Omega_{2}$, the Landau lower bound says that $D(M) \geq\left|\Omega_{1} \cup \Omega_{2}\right|$. Combining these two inequalities, we obtain $D(M)=\left|\Omega_{1} \cup \Omega_{2}\right|$ and hence $M$ achieves the Landau bound.

In summary, this lemma says that if two lattices are optimal (in the Landau sense) for two frequency regions, and their intersection is a sampling set for the intersection of the two frequency regions, then sampling on the union of these two lattices is optimal for the union of frequency regions. This simple result is powerful because it allows us to design new optimal sampling sets from other optimal sampling sets! This result can be applied to Proposition 2.2. If the original lattices $L_{1}$ and $L_{2}$ achieve the Landau bound for $\Omega_{1}$ and $\Omega_{2}$, respectively, and the conditions for Proposition 2.2 hold, then $M$ achieves the Landau bound for $\Omega$. Moreover, for each choice of $\Omega$ shown in Figure 2, if the original lattices $L_{1}$ and $L_{2}$ achieve the Landau bound for $\Omega_{1}$ and $\Omega_{2}$, respectively, then $M$ attains the Landau bound for $\Omega$.

\section{CONCLUSIONS AND FUTURE WORK}

Classical sampling results for sampling on a lattice $L_{i}$ specify conditions on a set $\Omega_{i}$ such that any field bandlimited to $\Omega_{i}$ can be reconstructed exactly from the measurements of the field on $L_{i}$. In this work, we presented sufficient conditions for perfectly reconstructing a field from a union of two lattices $L_{1}$ and $L_{2}$ that share a common lattice. In particular we focused on fields bandlimited to $\Omega=\Omega_{1} \cup \Omega_{2}$. These conditions can be used to reconstruct a wide array of frequency support regions, as summarized in Figure 2. Furthermore, a sufficient condition for achieving the Landau lower bound was given. There are many avenues for future work, including finding necessary conditions for perfect reconstruction besides the Landau bound, and generalizing these results to higher dimensions. For example, it may be possible to prove that the conditions of Proposition 2.4 are both necessary and sufficient to ensure that the union of lattices is a sampling set for spectra supported on the union $\Omega$ of the original spectra. Finally, we also believe that Proposition 2.4 can be generalized and expanded. 


\section{REFERENCES}

[1] M.G. Reyes, X. Zhao, D.L. Neuhoff, and T.N. Pappas, "Lossy compression of bilevel images based on markov random fields," in Proc. IEEE ICIP, San Antonio, Sept. 2007, vol. 2, pp. II-373.

[2] M.G. Reyes and D.L. Neuhoff, "Arithmetic encoding of markov random fields," in Proc. IEEE Int. Symp. Inform. Thy, Seoul, June 2009, pp. 532-536.

[3] M.G. Reyes and D.L. Neuhoff, "Lossless reduced cutset coding of markov random fields," in Proc. Data Compression Conf. 2010, pp. 386-395, Snowbird.

[4] A. Farmer, A. Josan, M.A. Prelee, D.L. Neuhoff, and T.N. Pappas, "Cutset sampling and reconstruction of images," in Proc. IEEE ICIP, Brussels, Sept. 2011, pp. 1909-1912.

[5] M.A. Prelee, D.L. Neuhoff, and T.N. Pappas, "Image reconstruction from a manhattan grid via piecewise plane fitting and gaussian markov random fields," in Proc. IEEE ICIP, Orlando, FL, Sept. 2012, pp. 2061-2064.

[6] M.A. Prelee and D.L. Neuhoff, "Performance-energy tradeoffs in cutset wireless sensor networks," in Proc. IEEE ICASSP, Florence, May 2014.

[7] M.A. Prelee and D.L. Neuhoff, "Energy efficient source localization on a manhattan grid wireless sensor network," in Proc. IEEE ICASSP, Vancouver, May 2013, pp. 4266-4270.

[8] M.A. Prelee and D.L. Neuhoff, "A sampling theorem for manhattan grids," in Proc. IEEE ICASSP, Kyoto, Mar. 2012, pp. 3797-3800.

[9] H.J. Landau, "Necessary density conditions for sampling and interpolation of certain entire functions," Acta Math., vol. 117, no. 1, pp. 37-52, 1967.

[10] D.P. Petersen and D. Middleton, "Sampling and reconstruction of wave-number-limited functions in n-dimensional euclidean spaces," Inf. Control, vol. 5, no. 4, pp. 279-323, 1962.

[11] A. Rosenfeld and A.C. Kak, Digital picture processing, Academic Press, New York, NY, 1976.

[12] A.M. Tekalp and A.M. Tekalp, Digital video processing, vol. 1, Prentice Hall PTR Upper Saddle river, NJ, 1995.

[13] D.E. Dudgeon and R.M. Mersereau, "Multidimensional digital signal processing," Prentice-Hall Signal Processing Series, Englewood Cliffs: Prentice-Hall, vol. 1, 1984.

[14] R. Venkataramani and Y. Bresler, "Perfect reconstruction formulas and bounds on aliasing error in sub-nyquist nonuniform sampling of multiband signals," IEEE Trans. Inform. Thy., vol. 46, no. 6, pp. 2173-2183, Sept. 2000.

[15] R. Venkataramani and Y. Bresler, "Optimal sub-nyquist nonuniform sampling and reconstruction for multiband signals," IEEE Trans. Inform. Thy, vol. 49, no. 10, pp. 23012313, Oct. 2001.

[16] Hamid Behmard and Adel Faridani, "Sampling of bandlimited functions on unions of shifted lattices," J. of Fourier Anal. Appl., vol. 8, no. 1, pp. 43-58, 2002.

[17] H. Behmard, "Efficient reconstruction algorithms using shifted lattices," IEEE Trans. Sig. Proc., vol. 57, no. 7, pp. 2548-2557, July 2009.
[18] J. Unnikrishnan and M. Vetterli, "Sampling high-dimensional bandlimited fields on low-dimensional manifolds," IEEE Trans. Info. Thy., vol. 59, no. 4, pp. 2103-2127, 2013.

[19] J. Unnikrishnan and M. Vetterli, "On sampling a highdimensional bandlimited field on a union of shifted lattices," in Proc. IEEE ISIT, 2012, pp. 1468-1472.

[20] J. Unnikrishnan and M. Vetterli, "Sampling and reconstruction of spatial fields using mobile sensors," IEEE Trans. Sig. Proc, vol. 61, no. 9, pp. 2328-2340, 2013. 